\title{
OPTIMAL SMOOTH PORTFOLIO SELECTION FOR AN INSIDER
}

\author{
YAOZHONG HU,* University of Kansas \\ BERNT ØKSENDAL, ${ }^{* *}$ University of Oslo and Norwegian School of \\ Economics and Business Administration
}

\begin{abstract}
We study the optimal portfolio problem for an insider, in the case where the performance is measured in terms of the logarithm of the terminal wealth minus a term measuring the roughness and the growth of the portfolio. We give explicit solutions in some cases. Our method uses stochastic calculus of forward integrals.
\end{abstract}

Keywords: Insider trading; optimal portfolio; enlargement of filtration; log utility; information flow

2000 Mathematics Subject Classification: Primary 60H40; 60H05; 60G15

Secondary 91B28

\section{Introduction}

There has been an increasing interest in the insider trading in recent years; see, for example, [1]-[6], [8]-[10], and the references therein. By an insider in a financial market we mean a certain investor who possesses more information than the information generated by the financial market itself. An insider may, for example, be an executive or simply an employee of a company. In probabilistic terminology, information is generally represented by a filtration. Usually an investor can only use the filtration generated by the market to make a decision. We call such investors honest. An insider has a larger filtration (more information) available to him and can use this larger filtration to make his decision; for example, to maximize his portfolio.

To simplify our presentation we assume that the market consists of the following two assets over the time period $[0, T]$. The first one is a bond whose price is determined by a stochastic process

$$
\mathrm{d} S_{0}(t)=r(t) S_{0}(t) \mathrm{d} t, \quad 0 \leq t \leq T .
$$

Another asset is the stock whose price follows the following geometric Brownian motion:

$$
\mathrm{d} S_{1}(t)=S_{1}(t)[\mu(t) \mathrm{d} t+\sigma(t) \mathrm{d} B(t)], \quad 0 \leq t \leq T,
$$

where $r(t), \mu(t)$, and $\sigma(t)$ are deterministic functions, $B(t)=B_{t}(\omega), 0 \leq t \leq T, \omega \in \Omega$, is a Brownian motion on a filtered probability space $\left(\Omega, \mathcal{F},\left\{\mathcal{F}_{t}\right\}_{t \geq 0}, \mathrm{P}\right)$, and $\mathrm{d} B(t)$ denotes the Itô-type stochastic differential. Denote the information generated by the market by $\widetilde{F}_{t}=$ $\sigma\left(B_{s}, 0 \leq s \leq t\right)$. Assume, for example, that at the beginning $(t=0)$ the insider knows, in addition to $\mathcal{F}_{t}$, the future value of the underlying Brownian motion at time $T_{0}$, where $T_{0}>T$.

Received 25 February 2005; revision received 24 July 2007.

* Postal address: Department of Mathematics, University of Kansas, 405 Snow Hall, Lawrence, KS 66045-2142, USA.

Email address: hu@math.ku.edu

** Postal address: Center of Mathematics for Applications (CMA), Department of Mathematics, University of Oslo, Box 1053 Blindern, Oslo, N-0316, Norway. Email address: oksendal@ math.uio.no 
Then his information filtration is given by $g_{t}=\sigma\left(B_{s}, 0 \leq s \leq t\right) \vee \sigma\left(B_{T_{0}}\right)$, the filtration generated by the Brownian motion up to time $t$ and $B_{T_{0}}$. The insider may use this filtration (rather than as usual using only the filtration $\mathscr{F}_{t}$ ) to optimize his portfolio.

More explicitly, let us express the portfolio in terms of the fraction $\pi(t)$ of the total wealth invested in the stocks at time $t$. Let $X^{(\pi)}(t)$ denote the corresponding wealth at time $t$. In [9], Pikovsky and Karatzas considered the problem of maximizing the expectation of the logarithmic utility of terminal wealth,

$$
\Phi_{g}:=\sup _{\pi}\left\{\mathrm{E}\left[\log \left(X^{(\pi)}(T)\right)\right]\right\}
$$

where the supremum is taken over all $g_{t}$-adapted portfolios $\pi(\cdot)$. They proved that in this case the optimal insider portfolio is

$$
\pi^{*}(t)=\frac{\mu(t)-r(t)}{\sigma^{2}(t)}+\frac{B\left(T_{0}\right)-B(t)}{\sigma(t)\left(T_{0}-t\right)} .
$$

Moreover, the corresponding maximal expected utility $\Phi_{g}$ is given by

$$
\Phi_{g}=\mathrm{E}\left[\int_{0}^{T}\left\{r(s)+\frac{1}{2} \frac{(\mu(s)-r(s))^{2}}{\sigma^{2}(s)}+\frac{1}{2\left(T_{0}-s\right)}\right\} \mathrm{d} s\right], \quad T_{0} \geq T .
$$

In particular, if $T_{0}=T$ we obtain

$$
\Phi_{g}=\infty
$$

This is clearly an unrealistic result. If $T_{0}=T$ we see, by (1.2), that the optimal portfolio $\pi^{*}$ needed to achieve $\Phi_{g}=\infty$ will converge towards the derivative of $B(t)$ at $t=T_{0}^{-}$. Thus, $\pi^{*}(t)$ will consist of more and more wild fluctuations as $t \rightarrow T_{0}^{-}$. This is both practically impossible and also undesirable from the point of view of the insider; he does not want to expose a too conspicuous portfolio, compared to that of the honest trader, which in the optimal case is just

$$
\pi_{\text {honest }}^{*}(t)=\frac{\mu(t)-r(t)}{\sigma^{2}(t)} .
$$

To model this constraint we propose to modify (1.1) in the following way.

Problem 1.1. Find $\pi^{*} \in \mathcal{A} g$ and $\Phi$ such that

$$
\begin{aligned}
\Phi & =\sup _{\pi \in \mathcal{A g}_{g}} \mathrm{E}\left[\log \left(X^{(\pi)}(T)\right)-\int_{0}^{T}|\mathbb{Q} \pi(s)|^{2} \mathrm{~d} s\right] \\
& =\mathrm{E}\left[\log \left(X^{\left(\pi^{*}\right)}(T)\right)-\int_{0}^{T}\left|\mathbb{Q} \pi^{*}(s)\right|^{2} \mathrm{~d} s\right],
\end{aligned}
$$

where $\mathcal{A g}_{\mathrm{g}}$ is a suitable family of admissible $g_{t}$-adapted portfolios $\pi$. Here, $\mathbb{Q}: \mathcal{A g}_{\mathrm{g}} \rightarrow \mathcal{A g}_{\mathrm{g}}$ is some linear operator measuring the size and/or the fluctuations of the portfolio. For example, we could have

$$
\mathbb{Q} \pi(s)=\lambda_{1}(s) \pi(s),
$$

where $\lambda_{1}(s) \geq 0$ is some given weight function. This models the situation where the insider is penalized for large volumes of trade.

An alternative choice of $\mathbb{Q}$ would be

$$
\mathbb{Q} \pi(s)=\lambda_{2}(s) \pi^{\prime}(s)
$$


for some weight function $\lambda_{2}(s) \geq 0$, where $\pi^{\prime}(s)=(\mathrm{d} / \mathrm{d} s) \pi(s)$. In this case, the insider is penalized for large trade fluctuations. Other choices of $\mathbb{Q}$ are also possible, including combinations of (1.3) and (1.4).

We will return to Problem 1.1 in Section 3, after giving a brief introduction to the forward integral.

\section{The forward integral}

In general, $B(t)$ need not be a semimartingale with respect to a bigger filtration $g_{t} \supset \mathcal{F}_{t}$. A simple example is

$$
g_{t}=\mathscr{F}_{t+\delta}, \quad t \geq 0,
$$

where $\delta>0$ is a constant.

Therefore, to be able to deal with corresponding (anticipating) $g_{t}$-adapted integrands $\phi(t, \omega)$, we must go beyond the semimartingale integral context. Following [3] we propose to use the forward integral to model such situations. This integral extends the semimartingale integral in the sense that the two integrals coincide if $B(t)$ is a semimartingale with respect to $g_{t}$.

In this section we briefly review some basic concepts and results on forward integrals. We refer to [3] for the motivation for using forward integrals in insider trading, and to [11] and [12] for more information about forward integrals.

Definition 2.1. ([11].) Let $\phi(t, \omega)$ be a measurable process (not necessarily adapted). Then the forward stochastic integral of $\phi$ is defined as

$$
\int_{0}^{\infty} \phi(t, \omega) \mathrm{d}^{-} B(t)=\lim _{\varepsilon \rightarrow 0} \int_{0}^{\infty} \phi(t, \omega) \frac{B(t+\varepsilon)-B(t)}{\varepsilon} \mathrm{d} t,
$$

if the convergence is in probability.

Let $\pi: 0=t_{0}<t_{1}<\cdots<t_{n}=t$ be a partition of $[0, T]$ and let $|\pi|=\max _{0 \leq j \leq n-1}\left(t_{j+1}-\right.$ $t_{j}$ ). It is easy to see that if $\phi$ is càdlàg then

$$
\int_{0}^{T} \phi(t, \omega) \mathrm{d}^{-} B(t)=\lim _{|\pi| \rightarrow 0} \sum_{j=0}^{n-1} \phi\left(t_{j}\right)\left(B\left(t_{j+1}\right)-B\left(t_{j}\right)\right) ;
$$

see [3] for details. Here $\mathrm{d}^{-} B(t)$ indicates that the integral is interpreted in the forward integral sense.

Definition 2.2. By a (1-dimensional) forward process we mean a process $X(t)=X(t, \omega)$ of the form

$$
X(t)=x+\int_{0}^{t} u(s, \omega) \mathrm{d} s+\int_{0}^{t} v(s, \omega) \mathrm{d}^{-} B(s), \quad t>0
$$

where $u(s, \omega)$ and $v(s, \omega)$ are measurable processes (not necessarily $\mathcal{F}_{t}$-adapted) such that

$$
\int_{0}^{t}|u(s, \omega)| \mathrm{d} s<\infty, \quad(\text { almost surely (a.s.)) for all } t>0,
$$

and the Itô forward integral

$$
\int_{0}^{t} v(s, \omega) \mathrm{d}^{-} B(s)
$$

exists for all $t>0$. 
In accordance with the classical Itô process notation, we use the short-hand notation

$$
\mathrm{d}^{-} X(t)=u(t) \mathrm{d} t+v(t) \mathrm{d}^{-} B(t)
$$

for the integral equation (2.2).

Theorem 2.1. ([12]; an Itô formula for forward processes.) Let

$$
\mathrm{d}^{-} X(t)=u(t) \mathrm{d} t+v(t) \mathrm{d}^{-} B(t)
$$

be a forward process. Let $f \in C^{2}(\mathbb{R})$ and define

$$
Y(t)=f(X(t)) .
$$

Then $Y(t)$ is also a forward process and

$$
\mathrm{d}^{-} Y(t)=f^{\prime}(X(t)) \mathrm{d}^{-} X(t)+\frac{1}{2} f^{\prime \prime}(X(t)) v^{2}(t) \mathrm{d} t .
$$

As an application of the Itô formula for forward integrals, we obtain the following result.

Corollary 2.1. ([3].) Let $u(t)$ and $v(t)$ be measurable processes such that the following integrals exist for all $t>0$ :

$$
\int_{0}^{t}\left(|u(s)|^{2}+|v(s)|^{2}\right) \mathrm{d} s, \quad \int_{0}^{t} v(s) \mathrm{d}^{-} B(s) .
$$

Then the forward stochastic differential equation

$$
\mathrm{d} X(t)=X(t)\left[u(t) \mathrm{d} t+v(t) \mathrm{d}^{-} B(t)\right], \quad X(0)=x>0,
$$

has the following unique solution:

$$
X(t)=x \exp \left(\int_{0}^{t}\left(u(s)-\frac{1}{2} v^{2}(s)\right) \mathrm{d} s+\int_{0}^{t} v(s) \mathrm{d}^{-} B(s)\right) .
$$

We also need the following result, which follows easily from Definition 2.1.

Lemma 2.1. Suppose that $\phi(t)$ is forward integrable and that $G$ is an $\mathcal{F}_{T}$-measurable random variable. Then we have

$$
\int_{0}^{T} G \phi(t) \mathrm{d}^{-} B(t)=G \int_{0}^{T} \phi(t) \mathrm{d}^{-} B(t) .
$$

\section{Optimal smooth portfolio for an insider}

We now return to Problem 1.1. We assume that the market consists of the following two investment possibilities:

(i) a bond, with price given by

$$
\mathrm{d} S_{0}(t)=r(t) S_{0}(t) \mathrm{d} t, \quad S_{0}(0)=1,0 \leq t \leq T,
$$

(ii) a stock, with price given by

$$
\mathrm{d} S_{1}(t)=S_{1}(t)[\mu(t) \mathrm{d} t+\sigma(t) \mathrm{d} B(t)], \quad 0 \leq t \leq T,
$$

where $T>0$ is constant and $r(t), \mu(t)$, and $\sigma(t)$ are given $\mathcal{F}_{t}$-adapted processes. 
We assume that

$$
\begin{gathered}
\mathrm{E}\left[\int_{0}^{T}\left\{|\mu(t)|+|r(t)|+\sigma^{2}(t)\right\} \mathrm{d} t\right]<\infty, \\
\sigma(t) \neq 0 \text { for almost all }(t, \omega) \in[0, T] \times \Omega .
\end{gathered}
$$

Let $g_{t} \supset \mathcal{F}_{t}$ be the information filtration available to the insider and let $\pi(t)$ be the portfolio chosen by the insider, measured in terms of the fraction of the total wealth $X(t)=X^{(\pi)}(t)$ invested in the stock at time $t \in[0, T]$. Then the corresponding wealth $X(t)=X^{(\pi)}(t)$ at time $t$ is modeled by the forward differential equation

$$
\begin{aligned}
\mathrm{d} X(t) & =(1-\pi(t)) X(t) r(t) \mathrm{d} t+\pi(t) X(t)\left[\mu(t) \mathrm{d} t+\sigma(t) \mathrm{d}^{-} B(t)\right] \\
& =X(t)\left[[r(t)+(\mu(t)-r(t)) \pi(t)] \mathrm{d} t+\sigma(t) \pi(t) \mathrm{d}^{-} B(t)\right] .
\end{aligned}
$$

For simplicity, we assume that $X(0)=1$. The motivation for using this forward integral model for the anticipating stochastic differential equation, (3.1), is the formula (2.1), which expresses the forward integral as a limit of Riemann sums of the Itô type, i.e. where the $i$ th term has the form $\phi\left(t_{i}\right)\left(B\left(t_{i+1}\right)-B\left(t_{i}\right)\right)$ with $\phi$ evaluated at the left end point $t_{i}$ of the interval $\left[t_{i}, t_{i+1}\right]$. Moreover, if $B(t)$ happens to be a semimartingale with respect to $g_{t}$ then the forward integral coincides with the semimartingale integral. See [3], [11], and [12] for more details on this.

We now specify the set $\mathcal{A}=\mathcal{A g}_{\mathrm{g}}$ of the admissible portfolios $\pi$ as follows.

Definition 3.1. In the following we let $\mathscr{A}=\mathcal{A g}$ denote a linear space of stochastic processes $\pi(t)$ such that (3.2)-(3.5) hold, where

$\pi(t)$ is $g_{t}$-adapted and the $\sigma$-algebra generated by

$$
\{\pi(t) ; \pi \in \mathcal{A}\} \text { is equal to } g_{t}, \text { for all } t \in[0, T],
$$

$\pi$ belongs to the domain of $\mathbb{Q}$,

$\sigma(t) \pi(t)$ is forward integrable,

$$
\mathrm{E}\left[\int_{0}^{T}|\mathbb{Q} \pi(t)|^{2} \mathrm{~d} t\right]<\infty .
$$

With these definitions we can now specify Problem 1.1 as follows.

Problem 3.1. Find $\Phi$ and $\pi^{*} \in \mathcal{A}$ such that

$$
\Phi=\sup _{\pi \in \mathcal{A}} J(\pi)=J\left(\pi^{*}\right),
$$

where

$$
J(\pi)=\mathrm{E}\left[\log \left(X^{(\pi)}(T)\right)-\frac{1}{2} \int_{0}^{T}|\mathbb{Q} \pi(s)|^{2} \mathrm{~d} s\right],
$$

$\mathbb{Q}: \mathcal{A} \rightarrow \mathcal{A}$ is a given linear operator, and $\mathrm{E}$ denotes the expectation with respect to $\mathrm{P}$. We call $\Phi$ the value of the insider and $\pi^{*} \in \mathcal{A}$ an optimal portfolio (if it exists). is

We now proceed to solve Problem 3.2. Using Corollary 2.4 we find that the solution to (3.1)

$$
X(t)=\exp \left(\int_{0}^{t}\left\{r(s)+(\mu(s)-r(s)) \pi(s)-\frac{1}{2} \sigma^{2}(s) \pi^{2}(s)\right\} \mathrm{d} s+\int_{0}^{t} \sigma(s) \pi(s) d^{-} B(s)\right) .
$$


Therefore, we obtain

$$
\begin{gathered}
J(\pi)=\mathrm{E}\left[\int_{0}^{T}\left\{r(t)+(\mu(t)-r(t)) \pi(t)-\frac{1}{2} \sigma^{2}(t) \pi^{2}(t)\right\} \mathrm{d} t\right. \\
\left.+\int_{0}^{T} \sigma(t) \pi(t) \mathrm{d}^{-} B(t)-\frac{1}{2} \int_{0}^{T}|\mathbb{Q} \pi(t)|^{2} \mathrm{~d} t\right] .
\end{gathered}
$$

To maximize $J(\pi)$ we use a calculus of variation technique as follows. Suppose that an optimal insider portfolio $\pi=\pi^{*}$ exists (in the following we omit the '*'). Let $\theta \in \mathcal{A}$ be another portfolio. Then the function

$$
f(y):=J(\pi+y \theta), \quad y \in \mathbb{R},
$$

is maximal for $y=0$; and hence,

$$
\begin{aligned}
& 0=f^{\prime}(0) \\
&=\frac{\mathrm{d}}{\mathrm{d} y}[J(\pi+y \theta)]_{y=0} \\
&=\mathrm{E}\left[\int_{0}^{T}\left\{(\mu(t)-r(t)) \theta(t)-\sigma^{2}(t) \pi(t) \theta(t)\right\} \mathrm{d} t\right. \\
&\left.\quad+\int_{0}^{T} \sigma(t) \theta(t) \mathrm{d}^{-} B(t)-\int_{0}^{T} \mathbb{Q} \pi(t) \mathbb{Q} \theta(t) \mathrm{d} t\right] .
\end{aligned}
$$

Let $\mathbb{Q}^{*}$ denote the adjoint of $\mathbb{Q}$ in the Hilbert space $L^{2}([0, T] \times \Omega)$, i.e.

$$
\mathrm{E}\left[\int_{0}^{T} \alpha(t)(\mathbb{Q} \beta)(t) \mathrm{d} t\right]=\mathrm{E}\left[\int_{0}^{T}\left(\mathbb{Q}^{*} \alpha\right)(t) \beta(t) \mathrm{d} t\right]
$$

for all $\alpha$ and $\beta$ in $\mathcal{A}$. Then we can rewrite (3.7) as

$$
\mathrm{E}\left[\int_{0}^{T}\left\{\mu(t)-r(t)-\sigma^{2}(t) \pi(t)-\mathbb{Q}^{*} \mathbb{Q} \pi(t)\right\} \theta(t) \mathrm{d} t+\int_{0}^{T} \sigma(t) \theta(t) \mathrm{d}^{-} B(t)\right]=0 .
$$

Now we apply this to a special choice of $\theta$. Fix $t \in[0, T]$ and $h>0$ such that $t+h<T$ and choose

$$
\theta(s)=\theta_{0}(t) \mathbf{1}_{[t, t+h]}(s), \quad s \in[0, T],
$$

where $\theta_{0}(t)$ is $g_{t}$-measurable. Then by Lemma 2.5 we have

$$
\begin{aligned}
\mathrm{E}\left[\int_{0}^{T} \sigma(s) \theta(s) \mathrm{d}^{-} B(s)\right] & =\mathrm{E}\left[\int_{t}^{t+h} \sigma(s) \theta_{0}(t) \mathrm{d}^{-} B(s)\right] \\
& =\mathrm{E}\left[\theta_{0}(t) \int_{t}^{t+h} \sigma(s) \mathrm{d} B(s)\right]
\end{aligned}
$$

Combining this with (3.8) we obtain

$$
\mathrm{E}\left[\left(\int_{t}^{t+h}\left\{\mu(s)-r(s)-\sigma^{2}(s) \pi(s)-\mathbb{Q}^{*} \mathbb{Q} \pi(s)\right\} \mathrm{d} s+\int_{t}^{t+h} \sigma(s) \mathrm{d} B(s)\right) \theta(t)\right]=0 .
$$


Since this holds for all such $\theta(t)$ we conclude that

$$
\mathrm{E}\left[M(t+h)-M(t) \mid g_{t}\right]=0,
$$

where

$$
M(t):=\int_{0}^{t}\left\{\mu(s)-r(s)-\sigma^{2}(s) \pi(s)-\mathrm{E}\left[\mathbb{Q}^{*} \mathbb{Q} \pi(s) \mid g_{s}\right]\right\} \mathrm{d} s+\int_{0}^{t} \sigma(s) \mathrm{d} B(s) .
$$

Since $\sigma \neq 0$ this proves the following result.

Theorem 3.1. Suppose that an optimal insider portfolio $\pi \in \mathcal{A}$ for Problem 3.2 exists. Then

$$
\mathrm{d} B(t)=\mathrm{d} \hat{B}(t)-\frac{1}{\sigma(t)}\left\{\mu(t)-\rho(t)-\sigma^{2}(t) \pi(t)-\mathrm{E}\left[\mathbb{Q}^{*} \mathbb{Q} \pi(t) \mid g_{t}\right]\right\} \mathrm{d} t,
$$

where $\hat{B}(t):=\int_{0}^{t} \sigma^{-1}(s) \mathrm{d} M(s)$ is a $g_{t}$-Brownian motion. In particular, $B(t)$ is a semimartingale with respect to $g_{t}$.

We now use this to find an equation for an optimal portfolio $\pi$.

Theorem 3.2. Assume that there exists a process $\gamma_{t}(s, \omega)$ such that $\gamma_{t}(s)$ is $g_{t}$-measurable for all $s \leq t$,

$$
t \rightarrow \int_{0}^{t} \gamma_{t}(s) \mathrm{d} s \quad \text { is of finite variation a.s. }
$$

and

$$
N(t):=B(t)-\int_{0}^{t} \gamma_{t}(s) \mathrm{d} s \text { is a martingale with respect to } g_{t} .
$$

Assume that $\pi \in \mathcal{A}$ is optimal, then

$$
\sigma^{2}(t) \pi(t)+\mathrm{E}\left[\mathbb{Q}^{*} \mathbb{Q} \pi(t) \mid g_{t}\right]=\mu(t)-r(t)+\sigma(t) \frac{\mathrm{d}}{\mathrm{d} t}\left(\int_{0}^{t} \gamma_{t}(s) \mathrm{d} s\right) .
$$

Proof. By comparing (3.9) and (3.10) we obtain

$$
\sigma(t) \mathrm{d} N(t)=\mathrm{d} M(t)
$$

i.e.

$$
-\sigma(t) \frac{\mathrm{d}}{\mathrm{d} t}\left(\int_{0}^{t} \gamma_{t}(s) \mathrm{d} s\right)=\mu(t)-r(t)-\sigma^{2}(t) \pi(t)-\mathrm{E}\left[\mathbb{Q}^{*} \mathbb{Q} \pi(t) \mid g_{t}\right] .
$$

Next we turn to a partial converse of Theorem 3.2.

Theorem 3.3. Suppose that (3.10) holds. Let $\pi(t)$ be a process solving (3.11). Suppose that $\pi \in \mathcal{A}$. Then $\pi$ is optimal for Problem 3.2.

Proof. Substituting

$$
\mathrm{d} B(t)=\mathrm{d} N(t)+\frac{\mathrm{d}}{\mathrm{d} t}\left(\int_{0}^{t} \gamma_{t}(s) \mathrm{d} s\right) \mathrm{d} t
$$

and

$$
\sigma(t) \pi(t) \mathrm{d}^{-} B(t)=\sigma(t) \pi(t) \mathrm{d} N(t)+\sigma(t) \pi(t) \frac{\mathrm{d}}{\mathrm{d} t}\left(\int_{0}^{t} \gamma_{t}(s) \mathrm{d} s\right) \mathrm{d} t
$$


into (3.6) we obtain

$$
\begin{aligned}
J(\pi)=\mathrm{E}\left[\int_{0}^{T}\{\right. & r(t)+(\mu(t)-r(t)) \pi(t)-\frac{1}{2} \sigma^{2}(t) \pi^{2}(t) \\
& \left.\left.+\sigma(t) \pi(t) \frac{\mathrm{d}}{\mathrm{d} t}\left(\int_{0}^{t} \gamma_{t}(s) \mathrm{d} s\right)-\frac{1}{2}|\mathbb{Q} \pi(t)|^{2}\right\} \mathrm{~d} t\right] .
\end{aligned}
$$

This is a concave functional of $\pi$, so if we can find $\pi=\pi^{*} \in \mathcal{A}$ such that

$$
\frac{\mathrm{d}}{\mathrm{d} y}\left[J\left(\pi^{*}+y \theta\right)\right]_{y=0}=0 \text { for all } \theta \in \mathcal{A},
$$

then $\pi^{*}$ is optimal. By a computation similar to the one leading to (3.8) we obtain

$$
\begin{aligned}
& \frac{\mathrm{d}}{\mathrm{d} y}\left[J\left(\pi^{*}+y \theta\right)\right]_{y=0} \\
& \quad=\mathrm{E}\left[\int_{0}^{T}\left\{\mu(t)-r(t)-\sigma^{2}(t) \pi^{*}(t)+\sigma(t) \frac{\mathrm{d}}{\mathrm{d} t} \int_{0}^{t} \gamma_{t}(s) \mathrm{d} s-\mathbb{Q}^{*} \mathbb{Q} \pi(t)\right\} \theta(t) \mathrm{d} t\right] .
\end{aligned}
$$

This is equal to 0 if $\pi=\pi^{*}$ solves (3.11).

We now apply this to some examples.

Example 3.1. Choose

$$
\mathbb{Q} \pi(t)=\lambda_{1}(t) \sigma(t) \pi(t)
$$

where $\lambda_{1}(t) \geq 0$ is deterministic.

Then (3.11) takes the form

$$
\sigma^{2}(t) \pi(t)+\lambda_{1}^{2}(t) \sigma^{2}(t) \pi(t)=\mu(t)-r(t)+\sigma(t) \frac{\mathrm{d}}{\mathrm{d} t} \int_{0}^{t} \gamma_{t}(s) \mathrm{d} s
$$

or

$$
\pi(t)=\pi^{*}(t)=\frac{\mu(t)-r(t)+\sigma(t)(\mathrm{d} / \mathrm{d} t) \int_{0}^{t} \gamma_{t}(s) \mathrm{d} s}{\sigma^{2}(t)\left[1+\lambda_{1}^{2}(t)\right]} .
$$

Substituting this into (3.12) we obtain the following result.

Theorem 3.4. Suppose that (3.10) and (3.13) hold. Let $\pi^{*}(t)$ be given by (3.14). If $\pi \in \mathcal{A}$ then $\pi^{*}$ is optimal for Problem 3.2. Moreover, the insider value is

$$
\begin{aligned}
\Phi & =J\left(\pi^{*}\right) \\
& =\mathrm{E}\left[\int_{0}^{T}\left\{r(t)+\frac{1}{2}\left(1+\lambda_{1}^{2}(t)\right)^{-1}\left(\frac{\mu(t)-r(t)}{\sigma(t)}+\frac{\mathrm{d}}{\mathrm{d} t} \int_{0}^{t} \gamma_{t}(s) \mathrm{d} s\right)^{2}\right\} \mathrm{d} t\right] .
\end{aligned}
$$

In particular, if we consider the case mentioned in Section 1, where

$$
g_{t}=\mathcal{F}_{t} \vee \sigma\left(B\left(T_{0}\right)\right) \quad \text { for some } T_{0}>T,
$$

then, by a result of Itô [7], we have

$$
\gamma_{t}(s)=\gamma(s)=\frac{B\left(T_{0}\right)-B(s)}{T_{0}-s},
$$


and (3.14) becomes

$$
\pi^{*}(t)=\sigma^{-2}(t)\left[1+\lambda_{1}^{2}(t)\right]^{-1}\left[\mu(t)-r(t)+\frac{\sigma(t)}{T_{0}-t}\left(B\left(T_{0}\right)-B(t)\right)\right] .
$$

The corresponding value is, by (3.15),

$$
J\left(\pi^{*}\right)=\mathrm{E}\left[\int_{0}^{T}\left\{r(t)+\frac{1}{2}\left(1+\lambda_{1}^{2}(t)\right)^{-1}\left(\frac{\mu(t)-r(t)}{\sigma(t)}+\frac{B\left(T_{0}\right)-B(t)}{T_{0}-t}\right)^{2}\right\} \mathrm{d} t\right] .
$$

In particular, we see that if $\sigma(t) \geq \sigma_{0}>0$ and

$$
\lambda_{1}(t)=\left(T_{0}-t\right)^{-\beta} \text { for some constant } \beta>0,
$$

then

$$
J\left(\pi^{*}\right) \leq C_{1}+C_{2} \int_{0}^{T}\left(T_{0}-t\right)^{-1+2 \beta} \mathrm{d} t<\infty
$$

for suitable constants $C_{1}$ and $C_{2}$, even if $T_{0}=T$. Thus, if we penalize large investments near $t=T_{0}$ then, according to (3.16), the insider obtains a finite value even if $T_{0}=T$.

Example 3.2. Next we put

$$
\mathbb{Q} \pi(t)=\pi^{\prime}(t) \quad\left(=\frac{\mathrm{d}}{\mathrm{d} t} \pi(t)\right) .
$$

This means that the insider is being penalized for large portfolio fluctuations. Choose $\mathcal{A}$ to be the set of all continuously differentiable processes $\pi(t)$ satisfying (3.2)-(3.5) and, in addition,

$$
\pi(0)=\pi(T)=0 \quad \text { a.s. }
$$

For simplicity, assume that

$$
\sigma(t) \equiv 1
$$

Then (3.11) can be expressed in the form

$$
\pi(t)-\pi^{\prime \prime}(t)=a(t)
$$

where

$$
a(t)=\mu(t)-r(t)+\frac{\mathrm{d}}{\mathrm{d} t}\left(\int_{0}^{t} \gamma_{t}(s) \mathrm{d} s\right) .
$$

Using the variation of parameter method we obtain the solution

$$
\pi(t)=\int_{0}^{t} \sinh (t-s) a(s) \mathrm{d} s+K \sinh (t),
$$

where, as usual, $\sinh (x)=\frac{1}{2}\left(\mathrm{e}^{x}-\mathrm{e}^{-x}\right), x \in \mathbb{R}$, is the hyperbolic sine function and the constant $K$ is chosen such that $\pi(T)=0$. In particular, if we again consider the case in which

$$
g_{t}=\mathcal{F}_{t} \vee \sigma\left(B\left(T_{0}\right)\right), \quad T_{0}>T,
$$

so that

$$
\gamma_{t}(s)=\gamma(s)=\frac{B\left(T_{0}\right)-B(s)}{T_{0}-s}, \quad 0 \leq s \leq T,
$$


then, by (3.19), we obtain

$$
\pi(t)=\int_{0}^{t} \sinh (t-s)\left[\mu(s)-r(s)+\frac{B\left(T_{0}\right)-B(s)}{T_{0}-s}\right] \mathrm{d} s+K \sinh (t) .
$$

By (3.12), the corresponding value is

$$
\begin{aligned}
J(\pi)=\mathrm{E}\left[\int _ { 0 } ^ { T } \left\{r(t)+(\mu(t)-r(t)) \pi(t)-\frac{1}{2} \pi^{2}(t)\right.\right. \\
\left.\left.\quad+\pi(t) \frac{B\left(T_{0}\right)-B(t)}{T_{0}-t}-\frac{1}{2}\left(\pi^{\prime}(t)\right)^{2}\right\} \mathrm{~d} t\right] .
\end{aligned}
$$

Note that if $0 \leq t \leq T<T_{0}$ then

$$
\begin{aligned}
\mathrm{E}\left[\pi(t) \frac{B\left(T_{0}\right)-B(t)}{T_{0}-t}\right] & \leq \mathrm{E}\left[\int_{0}^{t} \sinh (t-s) \frac{\left(B\left(T_{0}\right)-B(s)\right)\left(B\left(T_{0}\right)-B(t)\right)}{\left(T_{0}-s\right)\left(T_{0}-t\right)} \mathrm{d} s\right] \\
& =\int_{0}^{t} \frac{\sinh (t-s)}{T_{0}-s} \mathrm{~d} s .
\end{aligned}
$$

Therefore,

$$
J(\pi) \leq \int_{0}^{T}\left(\int_{0}^{t} \frac{\sinh (t-s)}{T_{0}-s} \mathrm{~d} s\right) \mathrm{d} t \leq \int_{0}^{T} \frac{\cosh (T-s)-1}{T-s} \mathrm{~d} s \text { for all } T_{0}>T .
$$

We have proved the following result.

Theorem 3.5. Suppose that $\mathbb{Q} \pi(t)=\pi^{\prime}(t)$ and $\mathcal{A}$ is chosen as in (3.17) and (3.18), and assume that $\sigma(t)=1$. Then the optimal insider portfolio is given by (3.19). In particular, if we choose

$$
\mathcal{g}_{t}=\mathcal{F}_{t} \vee \sigma\left(B\left(T_{0}\right)\right) \text { with } T_{0}>T,
$$

then the optimal portfolio $\pi$ is given by (3.20) and the corresponding insider value $J(\pi)$ is uniformly bounded for $T_{0}>T$.

Remark 3.1. Both Examples 3.6 and 3.8 yield ways to penalize the insider investor so that he would not obtain infinite utility. In Example 3.6, $\lambda_{1}(t)=\left(T_{0}-t\right)^{-\beta}$ for some $\beta>0$. To use this penalization, we need to know $T_{0}$. In Example 3.8, $T_{0}$ is not required to be known.

\section{Acknowledgement}

Y. Hu is supported in part by the National Science Foundation under grants DMS 0204613 and DMS 0504783.

\section{References}

[1] Amendinger, J., Imkeller, P. And Schweizer, M. (1998). Additional logarithmic utility of an insider. Stoch. Process. Appl. 75, 263-286.

[2] Bernardo, A. E. (2001). Contractual restrictions on insider trading: a welfare analysis. Computation and economic theory. Econom. Theory 18, 7-35.

[3] Biagini, F. And ØKsendal, B. (2005). A general stochastic calculus approach to insider trading. Appl. Math. Optimization 52, 167-181.

[4] Föllmer, H., Wu, C.-T. AND Yor, M. (1999). Canonical decomposition of linear transformations of two independent Brownian motions motivated by models of insider trading. Stoch. Process. Appl. 84, 137-164. 
[5] Huddart, S., Hughes, J. S. And Levine, C. B. (2001). Public disclosure and dissimulation of insider trades. Econometrica 69, 665-681.

[6] Imkeller, P., Pontier, M. And Weisz, F. (2001). Free lunch and arbitrage possibilities in a financial market model with an insider. Stoch. Process. Appl. 92, 103-130.

[7] ITô, K. (1978). Extension of stochastic integrals. In Proc. Internat. Symp. Stoch. Differential Equat., John Wiley, New York, pp. 95-109.

[8] Luo, S. AND Zhang, Q. (2002). Dynamic insider trading. In Applied Probability (Hong Kong, 1999; AMS/IP Stud. Adv. Math. 26), American Mathematical Society, Providence, RI, pp. 93-104.

[9] Pikovsky, I. and Karatzas, I. (1996). Anticipative portfolio optimization. Adv. Appl. Prob. 28, $1095-1122$.

[10] Postel-Vinay, F. ANd Zylberberg, A. (1997). Insiders et persistance: un réexamen dans un modèle de concurrence monopolistique. Ann. Économ. Statist. 1997, 161-181.

[11] Russo, F. And Vallois, P. (1993). Forward, backward and symmetric stochastic integration. Prob. Theory Relat. Fields 97, 403-421.

[12] Russo, F. AND Vallois, P. (2000). Stochastic calculus with respect to continuous finite quadratic variation processes. Stoch. Stoch. Reports 70, 1-40. 